\title{
Foodomics: a new comprehensive approach to food and nutrition
}

\author{
Francesco Capozzi $\cdot$ Alessandra Bordoni
}

Received: 31 July 2012/Accepted: 2 August 2012/Published online: 30 August 2012

(C) Springer-Verlag 2012

\begin{abstract}
In the past 20 years, the scientific community has faced a great development in different fields due to the development of high-throughput, omics technologies. Starting from the four major types of omics measurements (genomics, transcriptomics, proteomics, and metabolomics), a variety of omics subdisciplines (epigenomics, lipidomics, interactomics, metallomics, diseasomics, etc.) has emerged. Thanks to the omics approach, researchers are now facing the possibility of connecting food components, foods, the diet, the individual, the health, and the diseases, but this broad vision needs not only the application of advanced technologies, but mainly the ability of looking at the problem with a different approach, a "foodomics approach". Foodomics is the comprehensive, high-throughput approach for the exploitation of food science in the light of an improvement of human nutrition. Foodomics is a new approach to food and nutrition that studies the food domain as a whole with the nutrition domain to reach the main objective, the optimization of human health and well-being.
\end{abstract}

Keywords Foodomics - Omics technologies ·

Food science $\cdot$ Human nutrition

Human nutrition science has greatly developed in the past decades, turning from the consideration of foods as simply

Special section: "Foodomics"; Guest Editors Drs A. Bordoni and F. Capozzi

F. Capozzi · A. Bordoni $(\bowtie)$

Department of Food Sciences, University of Bologna,

Piazza Goidanich, 60, 47521 Cesena, FC, Italy

e-mail: alessandra.bordoni@unibo.it energy sources to the recognition of their role in maintaining health and in reducing the risk of diseases.

The importance of food for human health is not a new concept, considering Hippocrates's sentence "Let food be thy medicine and medicine be thy food"; the recent progresses in analytical methods allowed scientists to demonstrate the role of food in human health, and not to simply hypothesize it. So, according to another Hippocrates's sentence "There are in fact two things, science and opinion; the former begets knowledge, the latter ignorance", nowadays we are not simply thinking that a good diet is important for health, but we can demonstrate it, evidencing the mechanisms underlying these health effects.

In recent years, food science greatly grew as well, developing new food products, designing processes to produce these foods, improving packaging materials, food shelf-life, and sensory characteristics. Food chemistry, devoted to the evaluation of the molecular composition of food and the involvement of these molecules in chemical reactions, food physical chemistry, which studies both physical and chemical interactions in foods, and food microbiology also took great advantages from the new analytical methods.

Even though the readers of this journal are supposed to be more than familiar with these terms, let us introduce what we mean by the term "new analytical methods".

New analytical methods are mainly related to the holistic omics approach, implemented by "high-throughput" technologies. High-throughput refers to a technology in which a large (or even exhaustive) number of measurements can be taken in a fairly short time period. "Ome" and "omics" are suffixes that are derived from "genome", a term created by Hans Winkler in 1920, although the use of ome is older, signifying a homogeneous set of items as a whole.

Although four major types of high-throughput omics measurements are commonly performed (genomics, 
transcriptomics, proteomics, and metabolomics), a variety of omics sub-disciplines (epigenomics, lipidomics, interactomics, metallomics, diseasomics, etc.) have begun to emerge, each with their own set of instruments, techniques, reagents and software.

Transcriptomics, proteomics and metabolomics are all dynamic domains, as affected by interactions between the organism and external stimuli. The human being (the organism) can be affected by the diet (the external stimuli), that can intervene in a positive or negative way on the risk of occurrence of certain human diseases. But the diet is made of foods, and foods originate from living organisms, and can in turn be affected by external stimuli such as different agricultural and transforming technologies. Thus, to reach the final goal of improving human health, we need to consider all these possible dynamic interactions in an omics approach.

Thanks to the omics approach, researchers are now facing a new science which in theory can connect food components, foods, the diet, the individual, the health and the diseases. In practice, we are still far from this connection, which needs not only technologies but mainly a broad vision of the problem, since there are many actors playing the comedy. A broad vision means not only a broad expertise and the application of advanced technologies, but also the ability of looking at the problem with a different approach, a "foodomics approach".

Foodomics received the interest of scientists with different cultural background since 2009, at the time of the first international conference held in Cesena, Italy (foodomics.eu). The purpose of that conference was to promote a multidisciplinary environment where specialists in omics sciences were invited to contribute to the holistic definition of food and to trace a possible way to exploit this view in the nutrition field.

Food is a highly complex mixture and, with such complexity, its definition cannot be based only on preselected components. Moreover, its conventional definition, performed by a compositional analysis, is often affected by the extractive methods that, in turn, may not resemble the physiological environment where the molecules will become bioavailable. In fact, paying attention to particular components or methods, some chemical characteristics could be emphasized more than others, even if the latter may be more important to the food-human interaction point of view and to the health perspective. Equally, focusing our attention on the effect of specific nutrients on defined metabolic pathways we are at risk of forgetting the effect of the whole food on the whole human organism. To overcome this limit, the foodhuman interaction needs a higher definition for both sides, and foodomics responds to the challenge to achieve this aim.

Thus, foodomics is not the food science-related counterpart of the nutrition science-related nutrigenomics.
Foodomics is the comprehensive, high-throughput approach for the exploitation of food science in the light of an improvement of human nutrition.

Foodomics has been previously defined as a discipline that studies the Food and Nutrition domains through the application of omics technologies. In this context, nutrigenomics and nutrigenetics have been considered as a part of the more general foodomics term (Cifuentes 2009).

In our opinion, foodomics studies the food domain as a whole with the nutrition domain, applying the same advanced omics technologies to different samples, and integrates all results in order to have an overall vision allowing the improvement of health and well-being.

Indeed, food science and nutrition science can apply the same omics technologies to different samples. Besides the concept of biological sample in omics technology, already elucidated by Morrison et al. (2006), the same high-throughput analysis can be used in both food science and nutrition science. In human nutrition, genomics (the comprehensive analysis of DNA structure and function) is the scientific field of the genetic basis for the diverse responses to foods (and not the secret to personalizing diet and health; German et al. 2011); in food science it is the opportunity for improving our understanding of the history of plant domestication and to accelerate crop improvement (Morrell et al. 2011).

Transcriptomics allows to evidence the modulation of the global gene expression profile by different nutrients, correlating it to disease prevention (Bordoni et al. 2007), and to design microbial mitigation strategies for ready-toeat food products (Soni et al. 2011). Proteomics can greatly contribute to food safety (D'Alessandro and Zolla 2012) as well to the prevention of cancer by food bioactives (Shukla and George 2011). Last but not least, metabolomics, the systematic study of the unique chemical fingerprints that specific cellular processes leave behind, is rapidly becoming a fundamental approach in food science (Hong 2011) and in nutrition science as well (Puiggròs et al. 2011).

Technologies such as mass spectrometry (MS) or nuclear magnetic resonance (NMR) spectroscopy, can be used to characterize genetically modified crops (García-Cañas et al. 2011) as well as in studies aimed to improve health of individuals through diet (Rezzi et al. 2007), but they can also give new answers to old, unraveling questions such as food digestibility (Bordoni et al. 2011).

In this respect, also the traditional investigation techniques are experiencing a new age, since their integration with bio-informatics tools are putting a new light on the experimental data. The optimization of algorithms for genome-, proteome- and metabolome-wide statistical analyses are crucial tasks to exploit fully the potentials coming from the omics information. 
This holds particularly for NMR, which provides spectral data that could be considered, as a whole, the molecular profile of the sample and, for this, can mirror the whole metabolome present in the sample. Several applications of NMR metabonomics, recently appeared in literature, represent the opportunity to describe heterogeneity among food sources by looking at the effect of different production practices on the whole metabolic pattern, rather than focusing on specific metabolites (Savorani et al. 2010; Picone et al. 2011a, b).

The suitability of NMR metabonomics, as a general approach to evaluate the substantial chemical equivalence of wild type and GMOs grapes, emerges from a study which compared different cultivars of Vitis vinifera receiving a different number of the same exogenous gene, by looking at the changes occurring in the metabolic phenotype (metabotype; Picone et al. 2011a, b).

The increasing interest for the foodomics science encouraged the organizers to reiterate the same international conference on 2011, and four invited speakers are contributing with representative articles to the present special section hosted by Gene and Nutrition. Now, few words to introduce the contributions.

The article by Pérez-Massot et al. (2012) deals with the possibility of counteracting malnutrition by genetic engineering of staple crops, reviewing and discussing strategies for enhancing crop content of different nutrients. The authors also consider major constrains, that is, the risk assessments, that are at the basis of EU policies and regulation of GE crops. GE represents a "hot topic" not only in food science but also in human nutrition, and an omics view is surely needed to face all its implications.

The work by Valdés et al. (2012) describes the effect of rosemary extracts on colon cancer cells, and underlines the need of going behind the observational aspects, using omics approach such as transcriptomic profiling and functional enrichment analysis for elucidating signaling and metabolic pathways involved. This article highlights that all different aspects of Foodomics are, however, facing important challenges related to bioinformatics. Specialized bioinformatic platforms are essential to analyze and manage data in an efficient and reliable way.

The review authored by Cerdà and Manchado (2012) summarizes the use of comprehensive functional genomics, proteomics and metabolomics analyses aiming at better identifying the critical genes and molecules that control traits of commercial interest in aquaculture productions, such as growth rates, reproduction, larval development and disease resistance. An overview is given about the next-generation sequencing platforms which have drastically transformed the way researchers can address genomic questions on nonmodel fish species, allowing for a better optimization of production and management procedures in the industry.
Finally, the article by Montoliu et al. (2012) offers an overview on the opportunity offered by the determination of the system-wide (i.e., whole organism) biochemical effects of diets on individuals' metabolism, by unraveling the interactions in the complex mosaic of both genomic and meta-genomic (i.e., gut microbiota) network. The evolution of gene-metabolism researches, from singlegene/single-metabolite studies to genome wide association studies (GWAS), made it possible to perform unbiased searches of the entire human genome to identify all common genetic factors that affect the holistic metabolomics phenotypes.

In conclusion, given that the development and application of advanced omics methodologies and bioinformatics has contributed to the investigation of topics considered unapproachable few years ago, they represent the tools and not the scope, and the foodomics is much more than a further application of analytical methods. Foodomics is a new approach to food and nutrition, foodomics is a new science and a challenge to be faced leaving old concepts apart. In the foodomics field, researchers working in food chemistry, analytical chemistry, biochemistry, microbiology, molecular biology, food technology, clinical sciences, and human can finally work together, with an omics approach, to reach the main objective, the optimization of human health and well-being.

\section{Conflict of interest None.}

\section{References}

Bordoni A, Astolfi A, Morandi L, Pession A, Danesi F, Di Nunzio M, Franzoni M, Biagi P, Pession A (2007) N-3 PUFAs modulate global gene expression profile in cultured rat cardiomyocytes. Implications in cardiac hypertrophy and heart failure. FEBS Lett 581:923-929

Bordoni A, Picone G, Babini E, Vignali M, Danesi F, Valli V, Di Nunzio M, Laghi L, Capozzi F (2011) NMR comparison of in vitro digestion of Parmigiano Reggiano cheese aged 15 and 30 months. Magn Reson Chem 49:S61-S70

Cerdà J, Manchado M (2012) Advances in genomics for flatfish aquaculture. Gene Nutr. doi:10.1007/s12263-012-0312-8

Cifuentes A (2009) Food analysis and foodomics. J Chromatogr A 1216:7109

D'Alessandro A, Zolla L (2012) We are what we eat: food safety and proteomics. J Proteome Res 11:26-36

García-Cañas V, Simó C, León C, Ibáñez E, Cifuentes A (2011) MS-based analytical methodologies to characterize genetically modified crops. Mass Spectrom Rev 30:396-416

German JB, Zivkovic AM, Dallas DC, Smilowitz JT (2011) Nutrigenomics and personalized diets: what will they mean for food? Annu Rev Food Sci Technol 2:97-123

Hong YS (2011) NMR-based metabolomics in wine science. Magn Reson Chem 49(Suppl 1):S13-S21

Montoliu I, Genick U, Ledda M, Collino S, Martin F-P, le Coutre J, Rezzi S (2012) Current status on genome-metabolome wide associations: an opportunity in nutrition research. Gene Nutr (in press) 
Morrell PL, Buckler ES, Ross-Ibarra J (2011) Crop genomics: advances and applications. Nat Rev Genet 13:85-96

Morrison N, Cochrane G, Faruque N, Tatusova T, Tateno Y, Hancock D, Field D (2006) Concept of sample in OMICS technology. OMICS 10:127-137

Pérez-Massot E, Banakar R, Gómez-Galera S, Zorrilla-López U, Sanahuja G, Arjó G, Miralpeix B, Vamvaka E, Farré G, Rivera SM, Dashevskaya S, Berman J, Sabalza M, Yuan D, Bai C, Bassie L, Twyman RM, Capell T, Christou P, Zhu C (2012) The contribution of transgenic plants to better health through improved nutrition: opportunities and constraints. Gene Nutr (in press)

Picone G, Engelsen SB, Savorani F, Testi S, Badiani A, Capozzi F (2011a) Metabolomics as a powerful tool for molecular quality assessment of the fish Sparus aurata. Nutrients 3:212-227

Picone G, Mezzetti B, Babini E, Capocasa F, Placucci G, Capozzi F (2011b) Unsupervised principal component analysis of NMR metabolic profiles for the assessment of substantial equivalence of transgenic grapes (Vitis vinifera). J Agric Food Chem 59: 9271-9279

Puiggròs F, Solà R, Bladé C, Salvadó MJ, Arola L (2011) Nutritional biomarkers and foodomic methodologies for qualitative and quantitative analysis of bioactive ingredients in dietary intervention studies. J Chromatogr A 1218:7399-7414

Rezzi S, Ramadan Z, Fay LB, Kochhar S (2007) Nutritional metabonomics: applications and perspectives. J Proteome Res 6:513-525

Savorani F, Picone G, Badiani A, Fagioli P, Capozzi F, Engelsen SB (2010) Metabolic profiling and aquaculture differentiation of gilthead sea bream by H-1 NMR metabonomics. Food Chem 120:907-914

Shukla Y, George J (2011) Combinatorial strategies employing nutraceuticals for cancer development. Ann N Y Acad Sci 1229: $162-175$

Soni KA, Nannapaneni R, Tasara T (2011) The contribution of transcriptomic and proteomic analysis in elucidating stress adaptation responses of Listeria monocytogenes. Foodborne Pathog Dis 8:843-852

Valdés A, García-Cañas V, Rocamora-Reverte L, Gómez-Martínez A, Ferragut JA, Cifuentes A (2012) Effect of rosemary polyphenols on human colon cancer cells: transcriptomic profiling and functional enrichment analysis. Gene Nutr (in press) 